\section{Absorption of calcium and magnesium in patients with intestinal resections treated with medium chain fatty acids}

\author{
K V Haderslev, P B Jeppesen, P B Mortensen, M Staun
}

M Staun

Correspondence to: Dr K V Haderslev,

Department of Medical

Gastroenterology, CA 2121, Abdominal Centre,

Rigshospitalet, Blegdamsve

9, DK-2100 Copenhagen,

Denmark. Email:

khaderslev@dadlnet.dk

Accepted for publication 25 November 1999

Keywords: medium chain triglycerides; calcium absorption; magnesium absorption; intestinal resections; fat absorption

Patients with resections of the small bowel and malabsorption face several complications including the risk of skeletal demineralisation. ${ }^{1-3}$ The pathogenesis of bone disease is not fully understood but reduced absorption of vitamin $\mathrm{D},{ }^{4-6}$ dietary calcium, and magnesium ${ }^{378}$ are

\begin{abstract}
Department of Medical

Gastroenterology,

Abdominal Centre, Rigshospitalet, Copenhagen, Denmark

K V Haderslev

P B Jeppesen

P B Mortensen

Abstract

Background-Steatorrhoea is associated with increased faecal loss of calcium and magnesium. Medium chain C8-C10 triglycerides (MCTs) improve fat absorption in patients with small bowel resections but the effects on intestinal absorption of divalent cations are not clear.

Aim-To assess the effect of dietary replacement of long chain triglycerides (LCTs) with MCTs on calcium and magnesium absorption in patients with small bowel resections.

Patients-Nineteen adult patients with a remaining small intestine averaging 171 cm (range 50-300).

Methods-In a crossover design, patients were randomised to two high fat diets (10 $\mathrm{MJ} /$ day, $50 \%$ as fat) for four days each separated by one day of washout. Diets were prepared in duplicate and were based on either LCT (LCT period) or equal quantities of LCT and MCT (L/MCT period). Metabolic balances were calculated during the last three days of each period.

Results-Mean stool volume increased significantly with the L/MCT diet and was $336 \mathrm{ml}$ more than that with the LCT diet (95\% confidence interval of mean difference, 26-649 $\mathrm{ml}$ ). There was no significant change in the net absorption of calcium and magnesium between the two diets. On average, percentage calcium absorption was $8.6 \%$ with the LCT diet and $12.5 \%$ with the L/MCT diet. Mean percentage magnesium absorption was $5.4 \%$ with the LCT diet and $2.9 \%$ with the L/MCT diet. Conclusions-Dietary replacement of $50 \%$ long chain triglycerides with medium chain triglycerides in small bowel resected patients increased faecal volume significantly. No changes in the intestinal net absorption of calcium and magnesium were demonstrated.

(Gut 2000;46:819-823)
\end{abstract}

known to be important factors and may result in negative mineral balances with ensuing loss of bone mineral.

Malabsorption of fat may have particular implications for mineral absorption as previous studies have shown that a high excretion of fat is correlated with increased faecal losses of calcium and magnesium. ${ }^{9-11}$ This is generally thought to result from the precipitation of calcium and magnesium with fatty acids to form poorly absorbable soap complexes in the intestinal lumen. ${ }^{1{ }^{12}}$ In rodent studies it has been shown that the bioavailability of calcium from these insoluble soaps becomes less with increased chain length and with decreasing degree of unsaturation of the fatty acids. ${ }^{11} 1314$ Medium chain triglycerides (MCTs) (triglycerides of C8 and C10 fatty acids) have been shown to greatly reduce fat excretion in a variety of malabsorptive states, including the short bowel syndrome. ${ }^{15-17}$ Consequently, if there is a positive correlation between absorption of fat and divalent cations, a concomitant improvement in calcium and magnesium absorption would therefore be expected from dietary replacement of long chain triglycerides (LCTs) with MCTs. However, studies of the effect of MCTs on mineral absorption in adults with fat malabsorption are few and contradictory. Some have reported enhanced calcium absorption $^{15} 1819$ whereas others have reported no effect on calcium and magnesium absorption of MCT substitution. ${ }^{19-22}$

In addition to the possible beneficial effect of MCTs on the bioavailability of divalent cations, a hypothesis has been put forward that medium length triglycerides enhance calcium absorption because the calcium-fatty acid molecule can diffuse more easily through the paracellular spaces, especially the tight junction, than the free calcium ion. ${ }^{23}$ An effect of MCTs on the cellular mechanisms has been supported by experimental evidence in an animal model. Thus Devlin and colleagues ${ }^{24}$ demonstrated increased expression of messenger RNA encoding for the vitamin $\mathrm{D}$ dependent calcium binding protein calbindin $\mathrm{D}$ which plays a key role in transcellular active calcium transport.

Abbreviations used in this paper: MCT, medium chain triglycerides; LCT, long chain triglycerides; L/MCT, 50\% long chain triglycerides and 50\% medium chain triglycerides; BMI, body mass index; $\mathrm{BMD}$, bone mineral density; $\mathrm{Z}$ score, standard deviation score for bone mineral density (age, sex matched); T score, standard deviation score for bone mineral density (sex matched); CI, confidence interval. 
The effect of dietary replacement of $50 \%$ LCTs with MCTs on fat absorption in patients with steatorrhoea caused by small bowel resection has recently been investigated. ${ }^{25}$ Absorption of fat was significantly improved and we report findings on the effects of MCT substitution on calcium and magnesium absorption in these patients.

\section{Methods}

PATIENTS

Patients with a history of small bowel resection and a faecal energy loss in the range 2000$6000 \mathrm{~kJ} /$ day (approximately 500-1500 kcal/ day) on their habitual diet were considered as study participants. Patients who had undergone surgery within 12 months and those with inflammatory bowel disease showing signs of active inflammation were excluded. Twenty four patients were included in the study. Five patients were excluded during the study; three felt distaste for and were unable to eat the high fat diets as prescribed while two experienced abdominal discomfort. Thus nine men and 10 women completed the study (mean age 51 (SD 16) years; range 20-78). Mean body mass index (BMI) was calculated as $21.9(2.9) \mathrm{kg} / \mathrm{m}^{2}$ (range 16.0-24.8). Mean time elapsed since the last bowel resection was 7.4 (5.6) years (range 1.1-18). Diagnoses were Crohn's disease $(n=12)$, mesenteric infarctions $(n=3)$, and other gastrointestinal diseases $(n=4)$. Ten patients had small bowel resections with part of or the whole colon in continuity. Of these patients, three had the colon including the ileocaecal valve in continuity; three patients presented with an ileocaecal resection; and four had a right sided hemicolectomy. As judged from the surgical records, mean length of the residual small intestine in patients with the colon in continuity was $143(78) \mathrm{cm}$ (range 50-250). None of the patients had a colostomy. Nine patients had been colectomised in addition to the small bowel resections. Six of these patients presented with a jejunostomy $(<200 \mathrm{~cm}$ jejunum remnant), three patients with an ileostomy with ileal resections of 50, 50 , and $85 \mathrm{~cm}$, respectively. The length of the remaining small intestine in all 19 patients was on average $171(50) \mathrm{cm}$ (range 50-300).

For each patient, a standard deviation score ( $\mathrm{Z}$ score) for bone mineral density (BMD) at the hip and spine was determined by the Norland XR-36 DXA densitometer (Norland Corporation, Fort Atkinson, Wisconsin, USA) (table 1). The reference material for females consisted of 793 normal women who participated in the Copenhagen female study and the

Table 1 Measurements of bone mineral density by dual energy $x$ ray absorptiometry in 19 patients with small bowel resections. Values expressed as $Z$ and $T$ scores

\begin{tabular}{lllll}
\hline & Mean $(S D)$ & Range & $95 \%$ CI of the mean & p Value \\
\hline Hip & & & & \\
Z score & $-0.70(1.15)^{\star}$ & $-2.08 ; 2.87$ & $-1.25 ;-0.15$ & 0.01 \\
T score & $-1.62(1.19)$ & $-3.54 ; 1.54$ & $-2.19 ;-1.06$ & \\
Spine & & & $-1.13 ; 0.19$ & 0.14 \\
Z score & $-0.47(1.38)$ & $-2.61 ; 2.00$ & $-1.69 ;-0.53$ & \\
T score & $-1.11(1.22)$ & $-3.06 ; 1.01$ & -1.53
\end{tabular}

${ }^{\star}$ Significantly lower $\mathrm{Z}$ score in patients compared with the reference material, $\mathrm{p}<0.05$, Student's $t$ test. CI, confidence interval. normal values for men were supplied by Norland. In addition, $T$ scores were calculated according to the formula: $T$ score $=(($ measured $\mathrm{BMD})-($ mean maximal $\mathrm{BMD}$ of sex matched controls))/ (standard deviation of maximal BMD of sex matched controls). According to the criteria of the WHO, ${ }^{26}$ osteopenia and osteoporosis are defined as $1 \mathrm{SD}$ and $2.5 \mathrm{SD}$ below the maximal BMD of sex matched controls, respectively. The mean $\mathrm{Z}$ score of the hip was significantly reduced compared with the reference material $(p<0.05)$ but the mean spinal $Z$ score was not significantly reduced $(\mathrm{p}=0.14)$. Four patients $(26.3 \%)$ had osteoporosis and nine more patients $(52.6 \%)$ presented with osteopenia.

Six patients received long term parenteral nutrition or fluids supplying water, carbohydrates, amino acids, and electrolytes. Thus four patients without a colon received a mean volume of parenteral nutrition of 2.5 (1.6) litre/day (range 0.9-4.5), and two patients with a preserved colon received $2.2(0.5)$ litre/day (range 1.9-2.6) of parenteral nutrition. The mean intravenous cation supplementation in patients who received parenteral nutrition was 4.2 (3.5) $\mathrm{mmol}$ calcium/day (range 0-7.7) and 6.4 (3.6) $\mathrm{mmol}$ magnesium/day (range $0-10$ ). Of the remaining patients, 10 had peroral calcium supplementation of $13-39 \mathrm{mmol} /$ day and five patients received magnesium supplementation perorally containing $15-30 \mathrm{mmol} /$ day. Five patients received 1200 IU vitamin D intravenously once a week. Four patients had 100000 IU of vitamin D/month intramuscularly, and eight patients received vitamin D 400-1400 IU/day orally. Calcium, magnesium, and vitamin D supplementation was unchanged during the study.

STUDY PROTOCOL

The study protocol was approved by the Ethics Committee for Medical Research in Copenhagen, Denmark, and the study was conducted in accordance with the Declaration of Helsinki of 1975 , as revised in 1983. Informed consent was obtained from all patients before inclusion.

Two high fat diets (LCT and L/MCT) were designed from food tables to provide identical energy equivalents of 20:24:56\% carbohydrate: protein: fat with a total energy content obtained from dietary records. Duplicate diets were prepared for analysis. The LCT diet was based on ordinary dietary fat whereas half of the LCT (weight ratio) was replaced by MCT in the L/MCT diet by the use of MCT containing margarine (Van den Bergh Foods, Rotterdam, Netherlands), MCT oil, Triglydal (Laboratorie Sodietal, France), and an MCT oil emulsion, Liquigen (Nutricia/Scientific Hospital Supplies, Liverpool, UK).

Patients were admitted to the department for nine days. The diets were given for four days each in a randomised crossover design separated by a one day washout period. In each of the four day periods, faeces were collected for the last three days and urine for the last day. No transit marker was used for stool collection as the transit time in this particular group of patients had been measured within one year 
before the study and was found to be very short (mean 5.7 (SD 8.0) hours. Aliquots of diet and stools were collected on ice and immediately frozen at $-20^{\circ} \mathrm{C}$. Patients were allowed an unrestricted but recorded intake of no energy beverages; medications and parenteral supplements were kept constant. Mineral content of no energy beverages was obtained from dietary records.

ANALYSIS

Absorption of LCT, L/MCT, and total fat were based on the assumption that fat was ingested as the triglycerides but excreted as free fatty acids (that is, $1 \mathrm{~mol}$ of glycerol was added to every $3 \mathrm{~mol}$ of dietary fatty acids). Analysis was performed on homogenised and freeze dried aliquots of 24 hour pooled dietary and faecal samples. Fatty acids were determined by combined gas-liquid chromatography and mass spectrometry as previously described. ${ }^{25}$

The concentration of calcium and magnesium in aliquots of diet and stool homogenates was measured by atomic absorption spectrophotometry (Model 3100, Perkin Elmer, Connecticut, USA). The method of sample preparation differed between calcium and magnesium analyses. Thus dry ashed samples of homogenised diet and faeces redissolved in hydrochloric acid were analysed for calcium after dilution with lanthanum oxide/strontium chloride solution to eliminate phosphate interference. ${ }^{27-29}$ For magnesium analysis, the acid extraction method ${ }^{30}$ was preferred to the dry ash method ${ }^{29}$ as the latter yielded up to $50 \%$ lower concentrations, less recovery values, and considerable intra- and interassay variation. ${ }^{31}$ Thus acid extracted samples of diet and faeces were analysed for magnesium after dilution with a lanthanum oxide/strontium chloride solution. ${ }^{27} 28$ Measurements of calcium were performed in duplicate and the coefficient of variation was $4.6 \%$. Recovery of known amounts of calcium and magnesium added to the faeces was 95 (2)\% and 98 (3)\%, respectively (mean $(\mathrm{SD})$ ).

Net intestinal absorption was calculated by subtracting faecal excretion of calcium and magnesium from peroral intake. Thus the percentage absorption of each mineral was given as net intestinal absorption divided by per oral intake, expressed as a percentage.

STATISTICAL ANALYSIS

Results are expressed as mean (SD) and range. The Student's $t$ test for paired observations was used to compare changes between repeated measurements of paired variables. A p value $<0.05$ was considered statistically significant. The Microsoft EXCEL statistical program was used for all analyses.

\section{Results}

VOLUME AND FAT ABSORPTION

Data on intake, excretion, and percentage absorption of fat are summarised in table 2. As previously reported ${ }^{25}$ fat absorption expressed as a percentage of intake was significantly improved in patients on the L/MCT diet. On average fat absorption increased from $36 \%$ with the LCT diet to $56 \%$ with the L/MCT diet $(p=0.0003)$ even though fat intake was significantly higher in the L/MCT test period. Patients ingested identical amounts (weight) of food and drinks (weight) in the two study periods. Eleven of 19 patients experienced increased diarrhoea with the L/MCT diet and on average stool volume increased by $336 \mathrm{ml}$ $(p=0.04)$. The overall balances of fluids were equal between the two study periods; the larger stool volume with the L/MCT diet was counterbalanced by a tendency towards a lower urinary volume $(\mathrm{p}=0.06)$.

CALCIUM AND MAGNESIUM ABSORPTION

Calcium intake was similar in both test periods but there was a slight but significant difference in intakes of magnesium because of differences in the nutrient composition of the two diets (table 2). Magnesium and calcium absorption, expressed in absolute values and as a percentage of peroral intake, varied widely among patients during both dietary periods (fig 1). However, absorption of calcium and magnesium was independent of the quality of dietary fat as there was no significant difference

Table 2 Results of the metabolic balances in 19 patients with small bowel resection randomised and crossed over between the LCT and L/MCT diet

\begin{tabular}{|c|c|c|c|c|c|}
\hline & $L C T$ period & $L / M C T$ period & Diff. L/MCT-LCT & $95 \%$ CI of mean diff. & $p$ Value \\
\hline \multicolumn{6}{|l|}{ Fat (g/day) } \\
\hline Diet & $119(21)$ & $142(27)^{\star}$ & $24(21)$ & $14 ; 34$ & 0.0001 \\
\hline Faeces & $77(30)$ & $64(36)^{\star}$ & $-14(25)$ & $-26 ;-1$ & 0.03 \\
\hline Absorption (\%) & $36(18)$ & $56(21)^{\star}$ & $20(19)$ & $11 ; 29$ & 0.0003 \\
\hline \multicolumn{6}{|l|}{ Volume (kg/day) } \\
\hline Per oral & $4165(1019)$ & $4085(904)$ & $-80(306)$ & $-228 ; 67$ & 0.27 \\
\hline $\mathrm{PE}$ & 767 (1335) & 767 (1335) & & & \\
\hline Stool & $1548(1178)$ & $1883(1392)^{\star}$ & $336(643)$ & $26 ; 646$ & 0.04 \\
\hline Urine & 2259 (1318) & $1803(960)$ & $-456(993)$ & $-935 ; 22$ & 0.06 \\
\hline Balance & $1125(1055)$ & $1166(684)$ & $40(1010)$ & $-447 ; 527$ & 0.86 \\
\hline \multicolumn{6}{|l|}{ Calcium (mmol/day) } \\
\hline Per oral & $61.9(24.7)$ & $61.4(24.5)$ & $-0.6(5.6)$ & $-3.2 ; 2.1$ & 0.67 \\
\hline Stool & $56.7(29.8)$ & $53.0(23.8)$ & $-3.7(13.1)$ & $-10.0 ; 2.6$ & 0.24 \\
\hline Absorption & $5.3(12.9)$ & $8.4(12.1)$ & $3.1(15.4)$ & $-5.1 ; 11.3$ & 0.45 \\
\hline Absorption (\%) & $8.6(22.2)$ & $12.5(21.6)$ & $3.9(28.1)$ & $-9.6 ; 17.4$ & 0.55 \\
\hline \multicolumn{6}{|c|}{ Magnesium (mmol/day) } \\
\hline Per oral & $22.4(12.3)$ & $21.4(12.1)^{\star}$ & $-1.0(1.7)$ & $-1.8 ;-0.2$ & 0.02 \\
\hline Stool & $20.2(9.9)$ & $19.6(9.5)$ & $-0.5(5.7)$ & $-3.3 ; 2.2$ & 0.68 \\
\hline Absorption & $2.2(7.3)$ & $1.8(6.3)$ & $-0.4(6.2)$ & $-4.9 ; 4.1$ & 0.84 \\
\hline Absorption (\%) & $5.4(25.0)$ & $2.9(27.2)$ & $-2.5(29.6)$ & $-16.7 ; 11.8$ & 0.72 \\
\hline
\end{tabular}

${ }^{\star}$ Significantly different from LTC period: $\mathrm{p}<0.05$, Student's paired $t$ test.

PE, parenteral nutrition. 

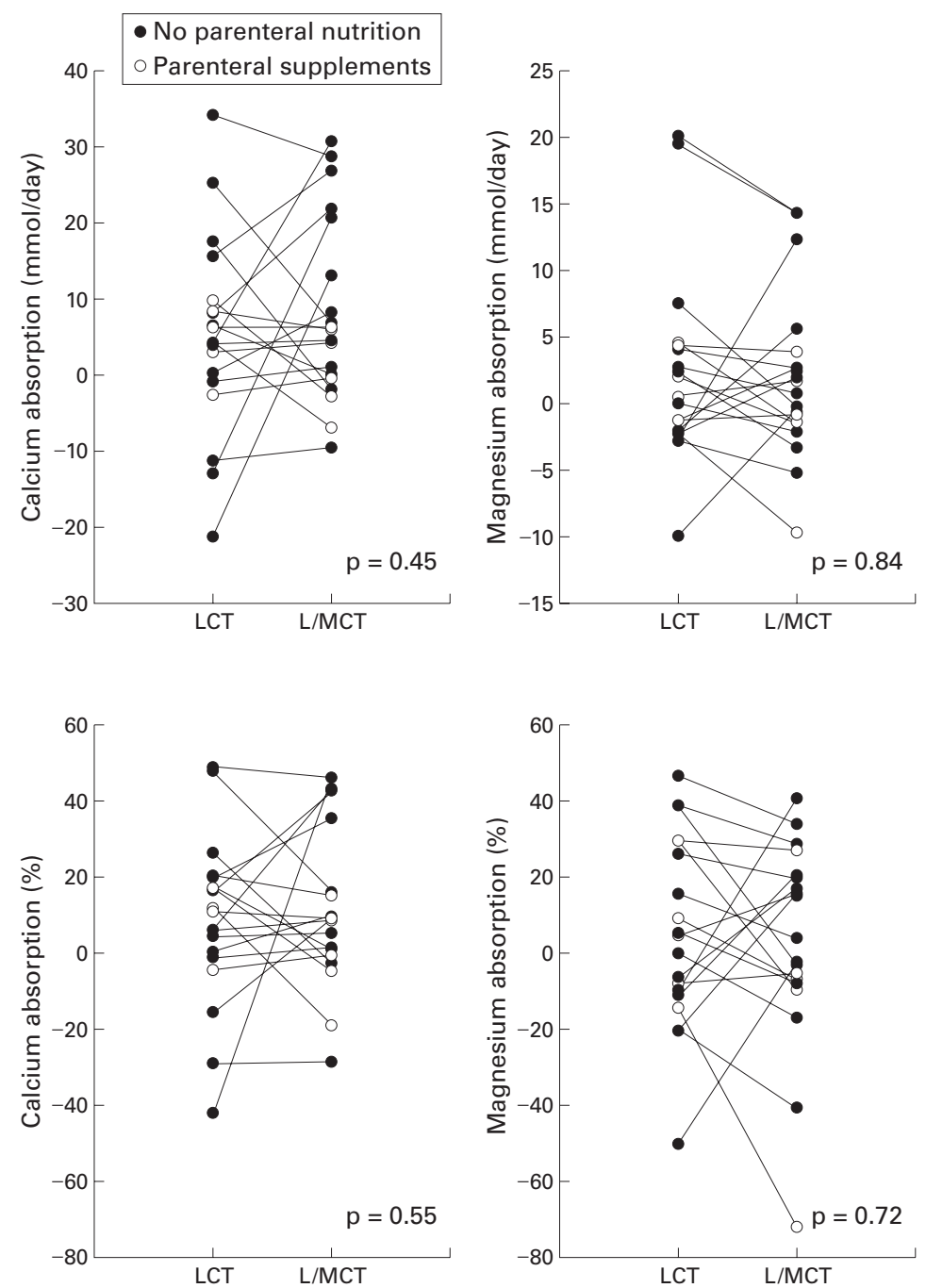

Figure 1 Absolute and percentage absorption of calcium and magnesium from the two diets. The LCT diet contained 100\% long chain triglycerides whereas the L/MCT diet had $50 \%$ long chain triglycerides and 50\% medium chain triglycerides. Each symbol indicates the result of one absorption study; results in the same patients are connected by lines.

between calcium and magnesium absorption during the two periods. Thus the mean net absorption of calcium was $5.3 \mathrm{mmol} /$ day (range -21.1 to 34.3 ) on the LCT diet with no significant change on the L/MCT diet $(\mathrm{p}=0.45)$. Correspondingly, magnesium absorption varied considerably with a mean value of $2.2 \mathrm{mmol} /$ day (range -20.1 to 10.0 ) with the LCT diet and no significant change on the $\mathrm{L} / \mathrm{MCT}$ diet $(\mathrm{p}=0.84)$. On average calcium absorption was $8.6 \%$ on the LCT diet and

Table 3 Differences in percentage absorption of calcium and magnesium with the L/MCT diet compared with the LCT diet in patients with and without a colon in continuity

\begin{tabular}{|c|c|c|c|}
\hline & Colon $(n=10)$ & No colon $(n=9)$ & $p$ Value \\
\hline \multicolumn{4}{|l|}{ Calcium } \\
\hline Absorption with LCT (\%) & $5.5(27.2)$ & $12.1(15.9)$ & \\
\hline Absorption with L/MCT (\%) & $9.2(23.6) \mathbb{S}$ & $16.2(19.8) \S$ & \\
\hline Diff. L/MCT $v$ LCT & $3.7(36.9)$ & $4.1(15.7)^{\star}$ & 0.82 \\
\hline \multicolumn{4}{|l|}{ Magnesium } \\
\hline Absorption with LCT (\%) & $5.8(30.9)$ & $4.9(18.3)$ & \\
\hline Absorption with L/MCT (\%) & $8.3(22.0) \sqrt{ }$ & $-3.1(32.2) \S$ & \\
\hline Diff. L/MCT $v$ LCT & $2.5(27.7)$ & $-8.0(32.3)^{\star}$ & 0.25 \\
\hline
\end{tabular}

$\S$ No significant change between LCT and L/MCT diets ( $\mathrm{p}>0.05$, Student's paired $t$ test). ${ }^{\star}$ No significant difference between patients with and without a colon in continuity $(p>0.05$, Student's $t$ test).
$12.5 \%$ on the L/MCT diet $(p=0.55)$. Conversely, the mean percentage of magnesium absorbed was $5.4 \%$ on the LCT diet and $2.9 \%$ on the L/MCT diet $(p=0.72)$. With the $\mathrm{L} / \mathrm{MCT}$ diet the percentage calcium absorption was improved in 10 patients and decreased in nine patients. The percentage magnesium absorption was improved in nine and had decreased in 10 patients. For patients with and without a colon in continuity, we found no difference in net percentage absorption of calcium or magnesium between the two diets (table 3). In addition, whether or not patients had a colon in continuity had no influence on the differences in the percentage absorption of calcium and magnesium with the L/MCT compared with the LCT diet.

Although net losses of calcium and magnesium were modest, two patients demonstrated massive calcium and magnesium losses irrespective of the dietary regimen.

\section{Discussion}

This study describes the effect of dietary MCTs on intestinal absorption of magnesium and calcium in small bowel resected patients. In view of the marked improvement in fat absorption with the L/MCT diet, we also anticipated increased mineral absorption. However, $50 \%$ replacement of LCTs with MCTs had no effect on net intestinal absorption of magnesium and calcium. Our results were based on metabolic balances generated in two three day study periods in which patients were randomised and crossed over between diets containing either LCT or L/MCT. This study design had the advantage that each patient served as his own control which eliminates errors caused by interindividual differences; the drawback is that theoretically there may have been a carryover of treatment effect from one period to the next, as only two days separated the two periods of stool collections. However, although we did not use a marker for faecal separation, we consider stool collections reliable, as the average transit time in the patients was less than six hours and none of the patients had a transit time exceeding 24 hours. Changes in the efficiency of absorption of calcium, in particular, is a major concern in balance studies as patients may adapt to the new diet with a gradual change in PTH and vitamin $\mathrm{D}$ levels over time. However, considering the relatively short test period it is unlikely that major adaptive changes occurred during the study.

Generally, MCTs are considered to reduce faecal losses of water and electrolytes in short bowel patients. ${ }^{15-1732}$ We cannot explain the increase in faecal volume with the L/MCT diet in our study but it may have resulted from an osmotic or secretory effect of the MCTs. In theory, the increased diarrhoea could result in a significantly higher loss of endogenously secreted cations thus accounting for the lack of improvement of MCT substitution on calcium and magnesium absorption.

The results of the previous studies on the effect of MCT treatment on intestinal absorption of divalent cations in humans are conflict- 
ing, and only few data are available on the influence of MCT substitution on calcium and magnesium absorption in patients with fat malabsorption. In 1966, Winawer and colleagues ${ }^{15}$ showed that MCT supplementation improved calcium absorption but only one patient with short bowel syndrome was studied. A beneficial effect of MCTs on calcium absorption was later demonstrated by Kehayoglou and colleagues ${ }^{18}$ in biliary cirrhosis and by Agnew and Holdsworth ${ }^{19}$ in patients with partial gastrectomy and in healthy subjects. However, Agnew and Holdsworth ${ }^{19}$ found no significant effect in patients with fat malabsorption due to coeliac disease, and Harrison and colleagues ${ }^{20}$ were unable to demonstrate any significant effect of MCTs on calcium absorption in patients with pancreatic insufficiency. The effect of MCTs on divalent cation absorption in patients with bowel resections has previously been investigated by Hofmann and Poley, ${ }^{21}$ who studied nine patients with ileal resection and various degrees of steatorrhoea, and by McIntyre and colleagues ${ }^{22}$ who studied seven patients with a high jejunostomy. In agreement with our data, they found no consistent effect of MCTs on either calcium or magnesium absorption. These inconsistencies regarding the effect of MCTs may be due to the differences in the mechanisms leading to calcium and magnesium malabsorption in various conditions. MCTs are included in the fat blends of several preterm feeding formulas to increase fat absorption and several studies have been undertaken to access the value of MCT supplementation on calcium and magnesium absorption in infants. In common with the studies in adults, results are conflicting. Some studies reported enhanced absorption $^{33-35}$ while others ${ }^{36}{ }^{37}$ concluded that MCTs had no significant effect on divalent cation absorption.

In summary, this study evaluated the effects of MCTs on calcium and magnesium absorption in small bowel resected patients. The main findings were that the L/MCT diet had no beneficial effect on the amount of calcium and magnesium absorbed and appeared to increase faecal volume significantly.

The technical assistance of Jette Christiansen, Anne Birgitte Larsen, Birthe Stenbæk Hansen, Kirsten Ahring, and AnneMarie Raabyemagle is greatly appreciated.

1 Compston JE, Ayers AB, Horton LW, et al. Osteomalacia after small-intestinal resection. Lancet 1978;1:9-12.

2 Compston JE, Horton LW, Laker MF, et al. Bone disease after jejuno-ileal bypass for obesity. Lancet 1978;2:1-4.

3 Hylander E, Ladefoged K, Madsen S. Calcium balance and bone mineral content following small-intestinal resection. Scand $\mathcal{F}$ Gastroenterol 1981;16:167-76.

4 Thompson GR, Lewis B, Booth CC. Absorption of vitamin D3-3H in control subjects and patients with intestinal malabsorption. $\mathcal{F}$ Clin Invest 1966;45:94-102.

5 Lo CW, Paris PW, Clemens TL, et al. Vitamin D absorption in healthy subjects and in patients with intestinal
malabsorption syndromes. Am f Clin Nutr 1985;42:644-9.

6 Leichtmann GA, Bengoa JM, Bolt MJ, et al. Intestinal absorption of cholecalciferol and 25-hydroxycholecalciferol in patients with both Crohn's disease and intestinal resecin patients with both Crohn' s disease

7 tion. Am f Clin Nutr 1991;54:548-52. Booth CC, MacIntyre I, Mollin DL. Nutritional problems
associated with extensive lesions of the distal small intestine associated with extensive lesions
in man. QfM 1964;33:401-20.
8 Krawitt EL, Beeken WL, Janney CD. Calcium absorption in Crohn's disease. Gastroenterology 1976;71:251-4.

9 Ovesen L, Chu R, Howard L. The influence of dietary fat on jejunostomy output in patients with severe short bowel syndrome. Am f Clin Nutr 1983;38:270-7.

10 Hessov I, Andersson H, Isaksson B. Effects of a low-fat diet on mineral absorption in small-bowel disease. Scand $\mathcal{F}$ Gastroenterol 1983;18:551-4.

11 Tadayyon B, Lutwak L. Interrelationship of triglycerides with calcium, magnesium and phosphorus in the rat. $\mathcal{F}$ Nutr 1969;97:246-54.

12 Steggerda FR, Mitchell HH. The calcium balance of adult human subjects on high- and low-fat (butter) diets. $\mathrm{F}$ Nutr 1951;45:201-11.

13 Gacs G, Barltrop D. Significance of Ca-soap formation for calcium absorption in the rat. Gut 1977;18:64-8.

14 Tadayyon B, Lutwak L. Effects of dietary triolein, tripalmiin, and L-phenylalanine on calcium absorption in the rat. Proc Soc Exp Biol Med 1969;130:978-9.

15 Winawer SJ, Broitman SA, Wolochow DA, et al. Successful management of massive small-bowel resection based on assessment of absorption defects and nutritional needs. $N$ Engl f Med 1966;274:72-8.

16 Bochenek W, Rodgers JB Jr, Balint JA. Effects of changes in dietary lipids on intestinal fluid loss in the short-bowel syndrome. Ann Intern Med 1970;72:205-13.

17 Tandon RK, Rodgers JB Jr, Balint JA. The effects of medium-chain triglycerides in the short bowel syndrome. Increased glucose and water transport. Am f Dig Dis 1972; 17:233-8.

18 Kehayoglou K, Hadziyannis S, Kostamis P, et al. The effect of medium-chain triglyceride on ${ }^{47}$ calcium absorption in patients with primary biliary cirrhosis. Gut 1973;14:653-6.

19 Agnew JE, Holdsworth CD. The effect of fat on calcium absorption from a mixed meal in normal subjects, patients with malabsorptive disease, and patients with a partial gastrectomy. Gut 1971;12:973-7.

20 Harrison JE, Mchattie JD, Ligon IR, et al. Effect of medium chain triglycerides on fecal calcium losses in pancreatic insufficiency. Clin Biochem 1973;6:136-40.

21 Hofmann AF, Poley RJ. Role of bile acid malabsorption in pathogenesis of diarrhea and steatorrhea in patients with ileal resection. I. Response to cholestyramine or replacement of dietary long chain triglyceride by medium chain triglyceride. Gastroenterology 1972;62:918-34.

22 McIntyre PB, Fitchew M, Lennard Jones JE. Patients with a high jejunostomy do not need a special diet. Gastroenterology 1986;91:25-33.

23 Bronner F. Calcium Absorption. In: Leonard RJ, ed. Physiology of the gastrointestinal tract, 2 nd edn. New York: Raven ology of the gastrointestinal
Press, 1987:1419-35.

24 Devlin A, Innis SM, Wall K, et al. Effect of medium-chain triglycerides on calbindin-D9k expression in the intestine. Lipids 1996;31:547-9.

25 Jeppesen PB, Mortensen PB. The influence of a preserved colon on the absorption of medium chain fat in patients with small bowel resection. Gut 1998;43:478-83.

26 WHO Study Group. Assessment of fracture risk and its application to screening for postmenopausal osteoporosis. WHO Technical Report Series, 843. Geneva: World Health Organization, 1994.

27 Gochman N, Givelber H. Automated, simultaneous microdetermination of calcium and magnesium by atomic determination of calcium and magnesi

28 Pybus J. Determination of calcium and magnesium in serum and urine by atomic absorption spectrophotometry. Clin Chim Acta 1969:23:309-17.

29 Gimblet EG, Marney AF, Bonsnes RW. Determination of calcium and magnesium in serum, urine, diet, and stool by atomic absorption spectrophotometry. Clin Chem 1967;13: 204-14

30 Ladefoged K. Determination of zinc in diet and faeces by acid extraction and atomic absorption spectrophotometry. Clin Chim Acta 1980;100:149-53.

31 Ladefoged K, Nicolaidou P, Jarnum S. Calcium, phosphorus, magnesium, zinc, and nitrogen balance in patients with severe short bowel syndrome. Am f Clin Nutr 1980;33: 2137-44.

32 Zurier RB, Campbell RG, Hashim SA, et al. Enrichment of depot fat with odd and even numbered medium chain fatty depot fat with odd and even number

33 Tantibhedhyangkul P, Hashim SA. Medium-chain triglyceride feeding in premature infants: effects on calcium and magnesium absorption. Pediatrics 1978;61:537-45.

34 Sulkers EJ, Lafeber HN, Degenhart HJ, et al. Comparison of two preterm formulas with or without addition of mediumchain triglycerides (MCTs). II: Effects on mineral balance. $\mathcal{f}$ Pediatr Gastroenterol Nutr 1992;15:42-7.

35 Shenai JP, Reynolds JW, Babson SG. Nutritional balance studies in very-low-birth-weight infants: enhanced nutrient retention rates by an experimental formula. Pediatrics 1980;66:233-8

36 Huston RK, Reynolds JW, Jensen C, et al. Nutrient and mineral retention and vitamin D absorption in low- birthweight infants: effect of medium-chain triglycerides. Pediatrics 1983;72:44-8.

37 Okamoto E, Muttart CR, Zucker CL, et al. Use of mediumchain triglycerides in feeding the low-birth-weight infant. Am $\mathcal{F}$ Dis Child 1982;136:428-31. 